\title{
Biogenic Synthesis of Silver Nanoparticles using Pomegranate Peel Polyphenols and Its Catalytic Action in Reduction of Acid Red 6
}

\author{
Ran Tao ${ }^{1, a}$, Ping Yao ${ }^{1, b^{*}}$, Jun Zhang ${ }^{1, c}$, Lei $X u^{1, d}$, Hui-Ying $W^{1, e}$, Mao-Mao \\ Ding ${ }^{1, f}$ and Xin-Sheng Zhu ${ }^{2, g}$ \\ ${ }^{1}$ Suzhou Institute of Trade and Commerce, 287 Xuefu Road, Suzhou City, Jiangsu Province \\ 215009, P.R. China \\ ${ }^{2}$ Faculty of Textile and Clothing Engineering, Soochow University, 178 Eastern Ganjiang Road, \\ Suzhou, Jiangsu 215021, P.R. China

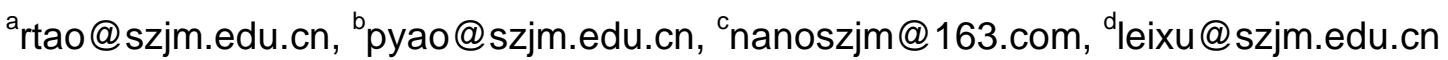 \\ ewhy_1220@sina.com, ${ }^{\mathrm{e}} 1377807526 @ q q . c o m,{ }^{\mathrm{g}}$ zhuxinsheng@suda.edu.cn
}

\begin{abstract}
Keywords: Biogenic synthesis, Pomegranate peel polyphenols, Silver nanoparticles, Catalytic activity, Acid Dye reduction

Abstract. A biogenic synthesis of silver nanoparticles (AgNPs) was achieved using pomegranate peel polyphenols in this article. The formation of AgNPs was confirmed by UV-vis spectrum and TEM image. The biogenic AgNPs showed Surface Plasmon Resonance (SPR) peak at 433nm as shown from the UV-vis absorption spectrum. The average diameter of AgNPs was around 26nm, which was testified by the particle size distribution analysis. The biogenic AgNPs were utilized for the catalytic reduction of Acid Red 6, and the green synthesized AgNPs showed high catalytic activity on Acid Red 6 reduction in the presence of $\mathrm{NaBH}_{4}$. The present study has brought to a novel method for the reduction of hazardous dyes in the liquid phase.
\end{abstract}

\section{Introduction}

The impact on human health due to environmental pollution has become the global concern. While the usage of noble metal nanoparticles especially silver nanoparticles (AgNPs) for the catalytic degradation of hazardous pollutants in the wastewater has aroused great attention ${ }^{[1-5]}$. In the same time, plant-mediated bioprocess provides an important opportunity for the green synthesis of AgNPs, and many kinds of plant extracts have been used for the synthesis of AgNPs ${ }^{[6-8]}$. Pomegranate peel polyphenols, as one kind of the plant extracts, has already used for the phytosynthesis of silver nanoparticles ${ }^{[9]}$. But, to the best knowledge of the authors, the catalytic reduction using pomegranate peel polyphenols synthesized AgNPs for the organic dyes has not been studied yet. In this study, AgNPs will be biogenic synthesized by pomegranate peel polyphenols, and the formation of AgNPs has been characterized by visible observation, UV-vis spectrophotometer, TEM and particle size distribution analysis. Furthermore, the catalytic activity of green synthesized AgNPs has be discussed by catalytic degradation of Acid Red 6 in the presence of $\mathrm{NaBH}_{4}$ in the aqueous solution.

\section{Materials and Methods}

\section{Materials}

$\mathrm{AgNO}_{3}(99.8 \%)$ and $\mathrm{NaBH}_{4}(98 \%)$ was purchased from Aladdin Industrial Corporation. pomegranate peel polyphenols (98\%) was purchased from Xi'An Bai Chuan Biotech Co., Ltd. The Aicd Red 6 dye was provided by the Zhejiang Runtu Co., Ltd. All the chemicals and reagents used in the paper were of analytical grade. 


\section{Synthesis and characterization of AgNPs}

$10 \mathrm{~mL}$ silver nitrate $(0.01 \mathrm{M})$ was added to $90 \mathrm{~mL}$ of freshly prepared pomegranate peel polyphenols solution in the ratio of 1:5. Progressively, formation of AgNPs was observed by visual color change to yellowish brown, which was further confirmed by UV-vis spectrophotometer.

\section{Characterization}

The reduction of silver ions was recorded by measuring the UV-visible absorption spectra of the liquid solutions in the beginning and after the synthesis reaction. The UV-visible absorption spectrum analysis was done by using UV-visible spectrophotometer (Shimadzu UV-3600) in the wavelength of 200-800nm. The size and morphology of AgNPs were determined by a TecnaiG220 (FEI, USA) at an accelerating voltage of 300kv. Particle size distribution of bio-reduced AgNPs in the aqueous solution was measured by the Zetasizer Nano series (Malven, UK) at $25^{\circ} \mathrm{C}$.

\section{Evaluation of catalytic activity of AgNPs}

Catalytic activity of synthesized AgNPs was performed by degradating Acid Red 6. 3mL Acid Red 6 $(50 \mathrm{mg} / \mathrm{L})$ solution was added with $0.1 \mathrm{~mL} \mathrm{NaBH}_{4}(0.2 \mathrm{M})$ and $0.1 \mathrm{ml} \mathrm{AgNPs}(100 \mu \mathrm{g} / \mathrm{mL})$ solution The reduction process was monitored by recording spectra using $\mathrm{UV}$-vis spectrophotometer at certain time intervals.

\section{Results and Discussion}

Upon the addition of silver nitrate to pomegranate peel polyphenols solution, the solution turned to yellowish brown after 30 mins reaction. This was indicated the formation of AgNPs ${ }^{[10]}$. The Surface Plasmon Resonance (SPR) peak appeared sharply at the wavelength of 433nm (Fig.1) for AgNPs, which was similar to the result by T.J.I. Edison ${ }^{[9]}$. The particle size distribution and morphology of AgNPs were characterized by Zetasizer Nano series and transmission electron microscope (TEM). As shown from Fig.2, the particle size of AgNPs distributed in two ranges of 1 10 nm and 10 100nm, and the average diameter of AgNPs was around $26 \mathrm{~nm}$ obtaining from the measuring result by the Zetasizer Nano series. The TEM image from Fig.3 also verified the great synthesis of AgNPs by pomegranate peel polyphenols, and TEM image also confirmed a typical spherical and an ellipsoidal morphology of AgNPs.

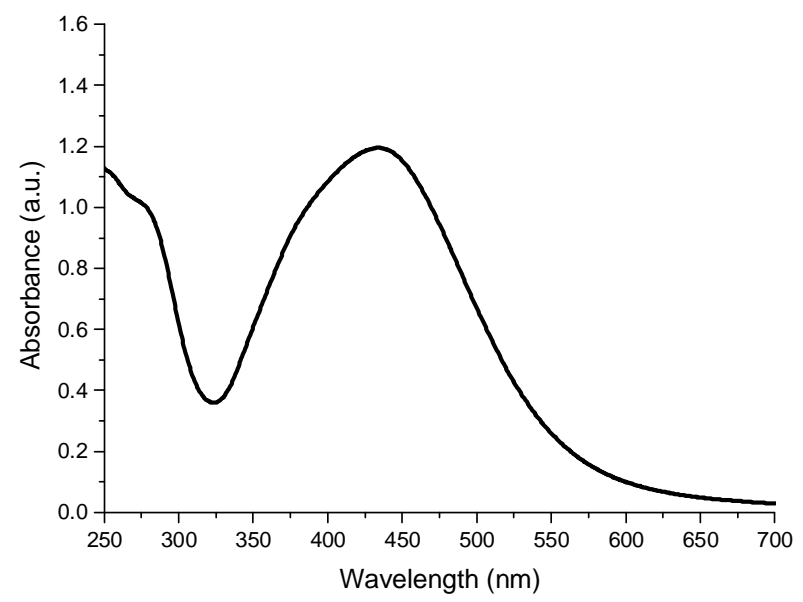

Fig.1 UV-Vis spectrum of biosynthesized AgNPs after 30 mins reaction.

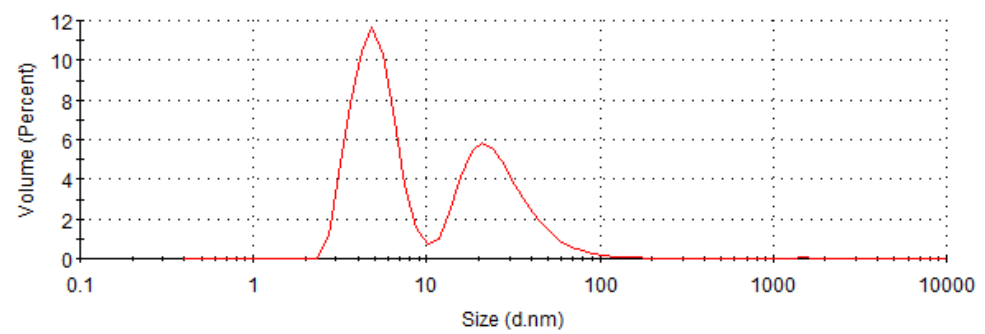

Fig.2 Particle size distribution of biosynthesized AgNPs. 


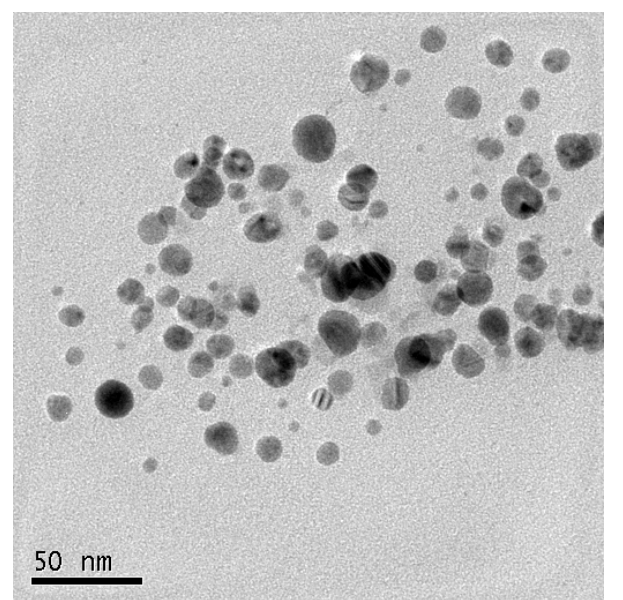

Fig.3 TEM image of biosynthesized AgNPs

AgNPs are well known for their catalytic activity and many reports are available on AgNPs as catalyst for the reduction/degradation of environmental pollutants such as 4-nitrophenol, $\mathrm{MB}$ and dyes [11]. The reduction of Acid Red 6 by $\mathrm{NaBH}_{4}$ was carried out using biogenic synthesized AgNPs as catalyst. The reduction process was monitored by using UV-Vis measurements at room temperature and the results were shown in Fig.4. As shown in Fig.4, the $\lambda_{\max }$ of Acid Red 6 was 525nm, and the intensity of the peak at $525 \mathrm{~nm}$ gradually decreased within 20mins. Experiments were also done for the reduction of Acid Red 6 by $\mathrm{NaBH}_{4}$ without of AgNPs (data was not shown), and there was no significant decrease after 40mins reaction. The results mentioned above demonstrated the great catalytic activity of biogenic synthesized AgNPs on the reduction of Acid Red 6 by $\mathrm{NaBH}_{4}$.

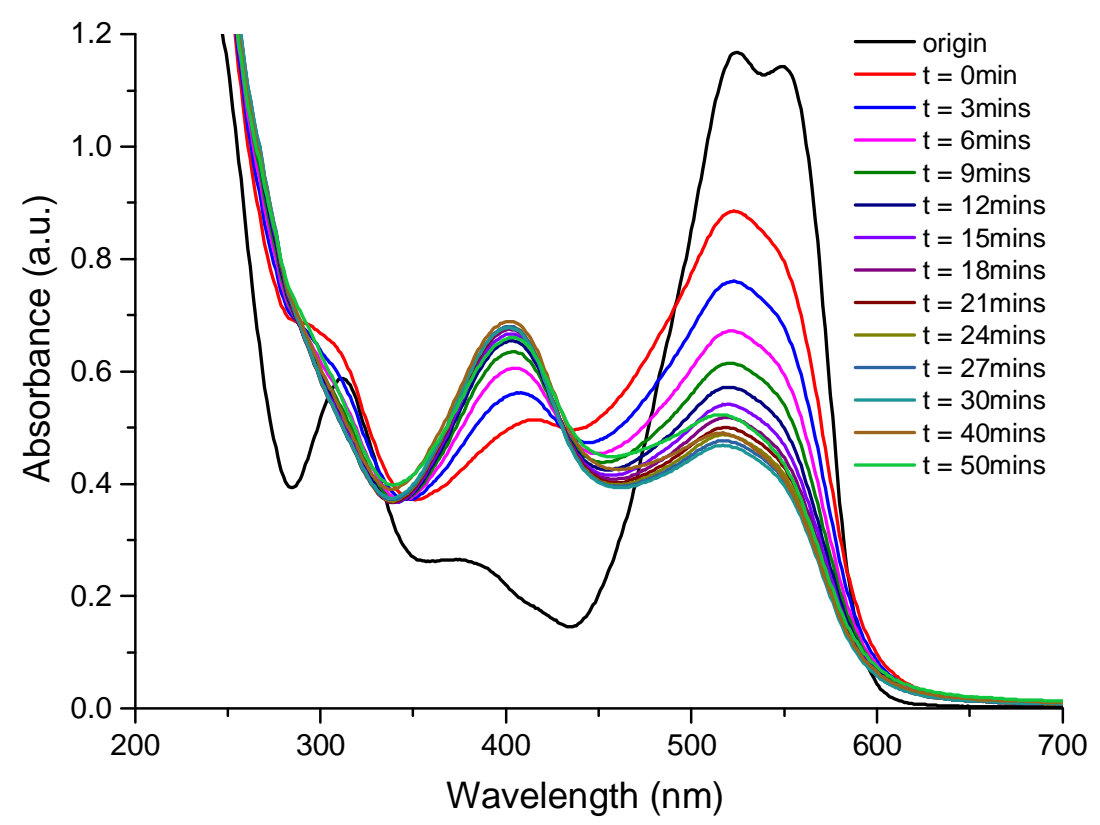

Fig.4 Reduction of Acid Red 6 by $\mathrm{NaBH}_{4}$ in the presence of AgNPs as catalyst.

\section{Conclusions}

The results from the study verified the possibility of biogenic synthesis of silver nanoparticles using pomegranate peel polyphenols. The biogenic AgNPs showed SPR peak at 433nm obtaining from the UV-vis absorption spectrum. The average diameter of AgNPs was around 26nm, which was testified by the particle size distribution analysis. The biogenic AgNPs showed high catalytic activity on reduction of Acid Red 6 by $\mathrm{NaBH}_{4}$. Thus the present study has brought to a novel method for the reduction of hazardous dyes using biogenic AgNPs. 


\section{Acknowledgements}

This work was supported by Jiangsu Natural Science Foundation of China (No. BK 20130344), National Special Foundation of Cocoon Silk Development Project 2015 (The Development and Industrialization of Digital Printing Composite Functional Silk Products), Science and Technology Guidance Project of China National Textile \& Apparel Council 2015 (No.2015060), and Middle and Young Academic Leaders of Blue Project 2014 (Jiangsu Provincial Education Department).

\section{References}

[1] Edison, T.J.I., Sethuraman, M. G.. Instant green synthesis of silver nanoparticles using Terminalia chebula fruit extract and evaluation of their catalytic activity on reduction of methylene blue. Process Biochemistry, Vol. 47(9) (2012) p. 1351-1357.

[2] Suvith, V. S., Philip, D. Catalytic degradation of methylene blue using biosynthesized gold and silver nanoparticles. Spectrochimica Acta - Part A: Molecular and Biomolecular Spectroscopy, Vol. 118, (2014) p.526-532.

[3] Wang Z., Xu C., Li X., Liu Z.. In situ green synthesis of Ag nanoparticles on tea polyphenolsmodified graphene and their catalytic reduction activity of 4-nitrophenol. Colloids and Surfaces A: Physicochemical and Engineering Aspects, Vol. 485, (2015) p. 102-110.

[4] Liu, Z., Yan, J., Miao, Y.-E., Huang, Y., \& Liu, T. Catalytic and antibacterial activities of green-synthesized silver nanoparticles on electrospun polystyrene nanofiber membranes using tea polyphenols. Composites Part B: Engineering, Vol. 79, (2015) p. 217-223.

[5] Ilunga, A. K., Meijboom, R.. Synthesis of narrowly dispersed silver and gold nanoparticles and their catalytic evaluation for morin oxidation. Applied Catalysis A: General, Vol. 509, (2016) p. 17-29.

[6] Rajan R., Chandran K., Harper S.L., Yun S.I., Kalaichelvan, P.T. Plant extract synthesized silver nanoparticles: An ongoing source of novel biocompatible materials. Industrial Crops and Products, 70, (2015) P. 356-373.

[7] Dhand V., Soumya L., Bharadwaj S., Chakra S., Bhatt D., Sreedhar B. Green synthesis of silver nanoparticles using Coffea arabica seed extract and its antibacterial activity. Materials Science and Engineering: C, Vol. 58, (2016) p. 36-43.

[8] Sinha T., Ahmaruzzaman M. High-value utilization of egg shell to synthesize Silver and Gold-Silver core shell nanoparticles and their application for the degradation of hazardous dyes from aqueous phase-A green approach. Journal of Colloid and Interface Science, Vol. 453, (2015) p. 115-131.

[9] T. Jebakumar Immanuel Edison, M.G. Sethuraman. Biogenic robust synthesis of silver nanoparticles using Punica granatum peel and its application as green catalyst for the reduction of an anthropogoenic pollutant 4-nitrophenol. Spectrochimica Acta Part A: Molecular and Biomolecular Spectroscopy. Vol. 104 (2013) p. 262-264.

[10] Pradnya Nalawade, Poulomi Mukherjee, Sudhir Kapoor. Spectrochimica Acta Part A: Molecular and Biomolecular Spectroscopy. Vol. 129 (14 August 2014), p. 121-124.

[11] Bogireddy N. K. R., Kiran Kumar H. A., Mandal, B. K. Biofabricated silver nanoparticles as green catalyst in the degradation of different textile dyes. Journal of Environmental Chemical Engineering, Vol 4(1) (2016) p. 56-64. 